\title{
Inclusive diffraction at HERA with a measured leading proton
}

\section{Florian Goebel* (on behalf of the $\mathbf{H 1}$ and ZEUS Collaborations)}

Address: DESY, Notkestr. 85, 22607 Hamburg, Germany

E-mail: 'f́goebel@mail. desy.dé

ABstract: Diffractive dissociation of virtual photons, $\gamma^{*} p \rightarrow X p$, has been studied in $e p$ interactions at HERA with the $\mathrm{H} 1$ and ZEUS detectors. The scattered proton is measured in spectrometers positioned downstream around the proton beampipe. The cross section is presented as a function of the four-momentum transfer squared $t$ and the azimuthal angle between the electron scattering plane and the proton scattering plane. The data are analyzed in terms of the diffractive structure function $F_{2}^{D(3)}\left(x_{I P}, \beta, Q^{2}\right)$.

\section{Introduction}

The diffractive dissociation of real photons, $\gamma p \rightarrow X p$, has characteristics similar to those of the dissociation of hadrons and can be described by Regge phenomenology. Within this framework, diffractive reactions at high energy are dominated by the exchange of a trajectory with the quantum numbers of the vacuum, referred to as the Pomeron trajectory. HERA allows the investigation of the partonic nature of diffraction in deep-inelastic scattering (DIS) using virtual photons as a probes. It is expected that the diffractive dissociation of virtual photons with high virtualities $Q^{2}$ can be described in terms of perturbative QCD.

In diffractive DIS, $e p \rightarrow e X N$, the photon dissociation system $X$ with mass $M_{X}$ is usually reconstructed in the central detector and is well separated in rapidity from the proton remnant system $N$ which, for small masses $M_{N}$, escapes through the forward beam hole of the detector. Diffractive events have been selected either by requiring an absence of particles in the forward region of the central detector (large rapidity gap method) [i] or by making use of the characteristic distribution of the mass $M_{X}$. However, for the analyses presented here, the scattered proton is measured in the $\mathrm{H} 1$ forward proton spectrometer (FPS) or the ZEUS leading proton spectrometer (LPS). The detection of the final state proton not only allows the measurement of its momentum but also provides the cleanest selection of diffractive events, $e p \rightarrow e X p$, independent of the hadronic final state and free from proton dissociation background.

\footnotetext{
${ }^{*}$ Speaker.
} 


\section{Analyses}

Protons scattered at small angles are deflected by the magnets of the beam optics into a system of detectors which are placed close to the proton beam to measure the fourmomentum of the scattered proton. The H1 FPS consists of four stations of scintillating fiber hodoscopes installed between $64 \mathrm{~m}$ and $90 \mathrm{~m}$ from the interaction point. The ZEUS LPS is a set of six silicon microstrip stations situated $24 \mathrm{~m}$ to $90 \mathrm{~m}$ downstream of the interaction point. Diffractive DIS events are selected by requiring a reconstructed positron in the central detector and a measured leading proton in the FPS or LPS. The kinematic variables $Q^{2}, x_{I P}, \beta$ and $t$ are reconstructed from the scattered positron, the hadronic final state measured in the central detector and the scattered proton. The H1 analysis is based on a luminosity of $28.8 \mathrm{pb}^{-1}$ and is restricted to the kinematic range $2<Q^{2}<50 \mathrm{GeV}^{2}$, $x_{I P}<0.09$ and $0.08<|t|<0.45 \mathrm{GeV}^{2}$ while the ZEUS analysis uses $12.8 \mathrm{pb}^{-1}$ and is restricted to $4<Q^{2}<100 \mathrm{GeV}^{2}, x_{\mathbb{P}}<0.03$ and $0.075<|t|<0.3 \mathrm{GeV}^{2}$.

\section{Results}

\section{$3.1 t$-dependence}
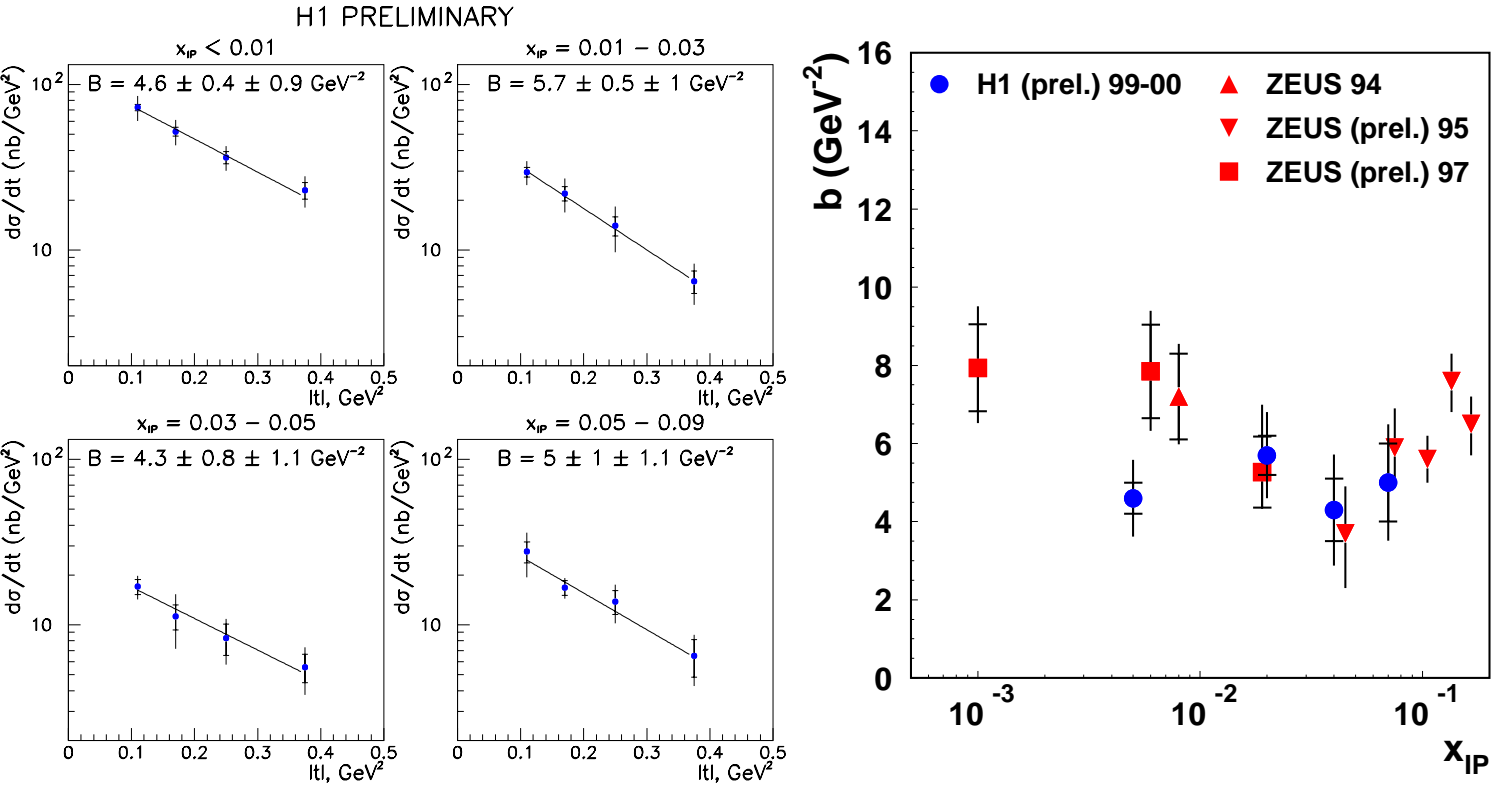

Figure 1: Left: The differential cross section $d \sigma / d t$ measured with the H1 FPS in four different $x_{I P}$ bins. Results of the fit with a function $d \sigma / d t \propto e^{b t}$ are shown. Right: The slope parameter $\mathrm{b}$ is plotted as a function of $x_{I P}$. Results obtained with the H1 FPS and the ZEUS LPS are shown.

A typical feature of diffractive events is an exponential fall of the differential cross section with $|t|$ at small values of $|t|$. In Fig. 1', the cross section is parameterized as $d \sigma / d t \propto e^{b|t|}$ in bins of $x_{I P}$, and the slope parameter $b$ is plotted as a function of $x_{I P}$. The results of $\mathrm{H} 1[\overline{2}]$ and ZEUS [3ij] are consistent within the experimental uncertainties and no significant dependence on $x_{I P}$ is visible. 


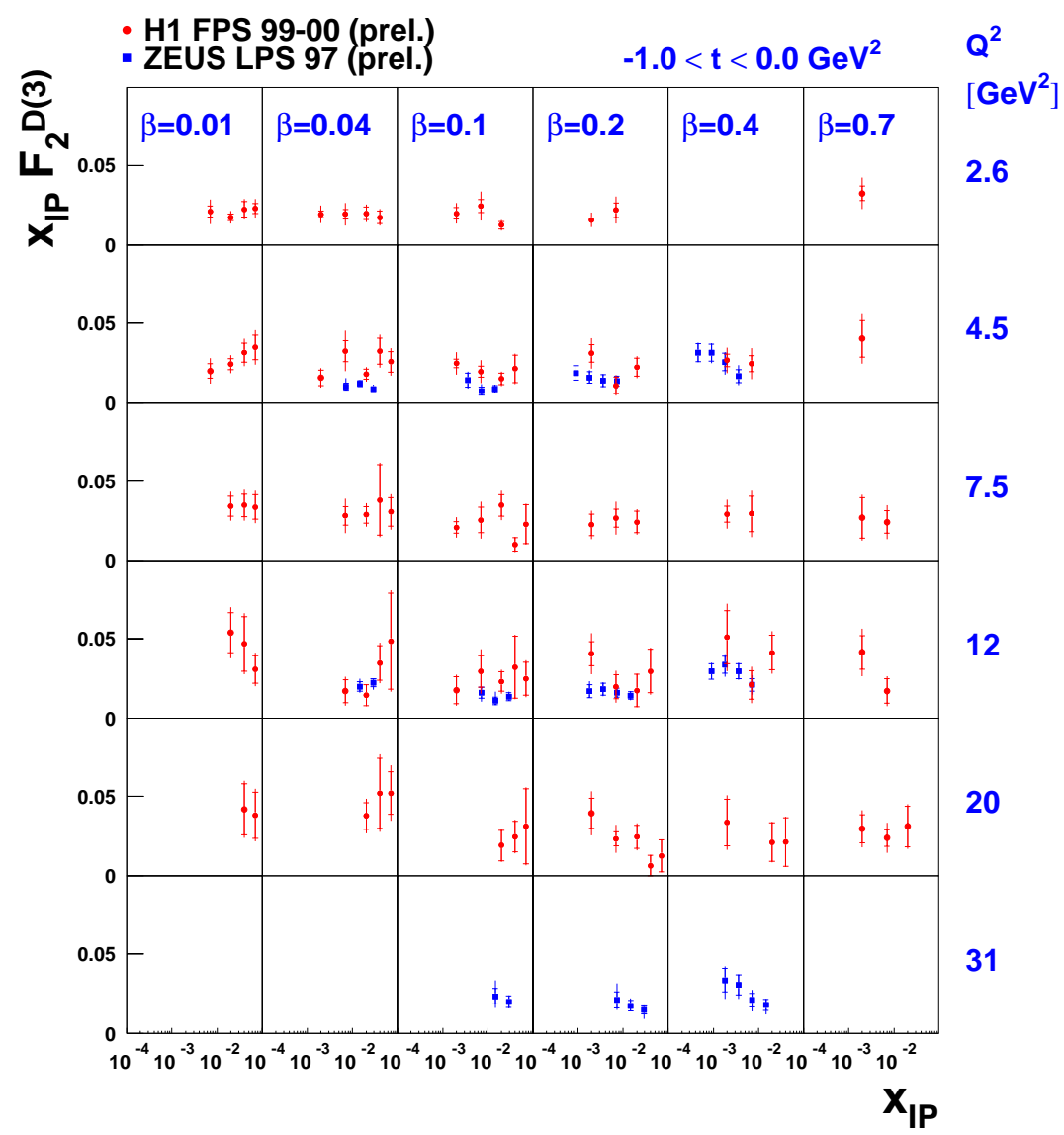

Figure 2: $\quad$ The diffractive structure function $x_{I P} F_{2}^{D(3)}$ as obtained by $\mathrm{H} 1$ and ZEUS is plotted as a function of $x_{I P}$ in bins of $\beta$ and $Q^{2}$.

\subsection{Diffractive structure function}

The diffractive cross section for the process $e p \rightarrow e X p$ can be expressed in terms of the diffractive structure function $F_{2}^{D(3)}$ as $\frac{d \sigma^{D}}{d \beta d Q^{2} d x_{I P}}=\frac{2 \pi \alpha^{2}}{\beta Q^{4}}\left[1+(1-y)^{2}\right] F_{2}^{D(3)}\left(\beta, Q^{2}, x_{I P}\right)$, where $x_{I P}=\left(Q^{2}+M_{X}^{2}\right) /\left(Q^{2}+W^{2}\right)$ is the fraction of the proton momentum carried by the Pomeron and $\beta=Q^{2} /\left(Q^{2}+M_{X}^{2}\right)$ is the fraction of the Pomeron momentum carried by the

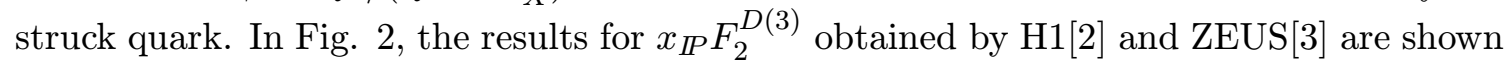
as a function of $x_{I P}$. The data are extrapolated to the full $t$ range using the $t$ dependence measured by each experiment. Good agreement is observed within uncertainties.

In Fig. $13 i$, the values of $x_{I P} F_{2}^{D(3)}$ obtained with the requirement of a leading proton are compared to the recent $\mathrm{H} 1$ measurement $\left[\begin{array}{l}\overline{4} \\ \mathbf{i}\end{array}\right]$ using a large rapidity gap in the central detector. Within uncertainties, the leading proton data are in good agreement with the rapidity gap data indicating that the contribution of proton dissociation to the latter analysis is small.

In factorisable models, $F_{2}^{D(3)}$ factorises into a Pomeron flux $f_{I P}\left(x_{I P}\right)$ and the structure function $F_{2}^{D(2)}\left(\beta, Q^{2}\right)$. Assuming a Regge-inspired power law dependence of $f_{I P}\left(x_{I P}\right) \propto$ $\left(1 / x_{I P}\right)^{2 \alpha_{I P}(t)-1}$, ZEUS found consistency with factorization and obtained $\alpha_{I P}(0)=1.13 \pm$ $0.03(\text { stat })_{-0.01}^{+0.03}$ (syst). In Fig. ${ }_{-0}^{-1} F_{2}^{D(2)}$ is shown as a function of $\beta$ and $Q^{2}$. Clear scaling

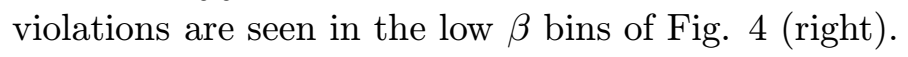




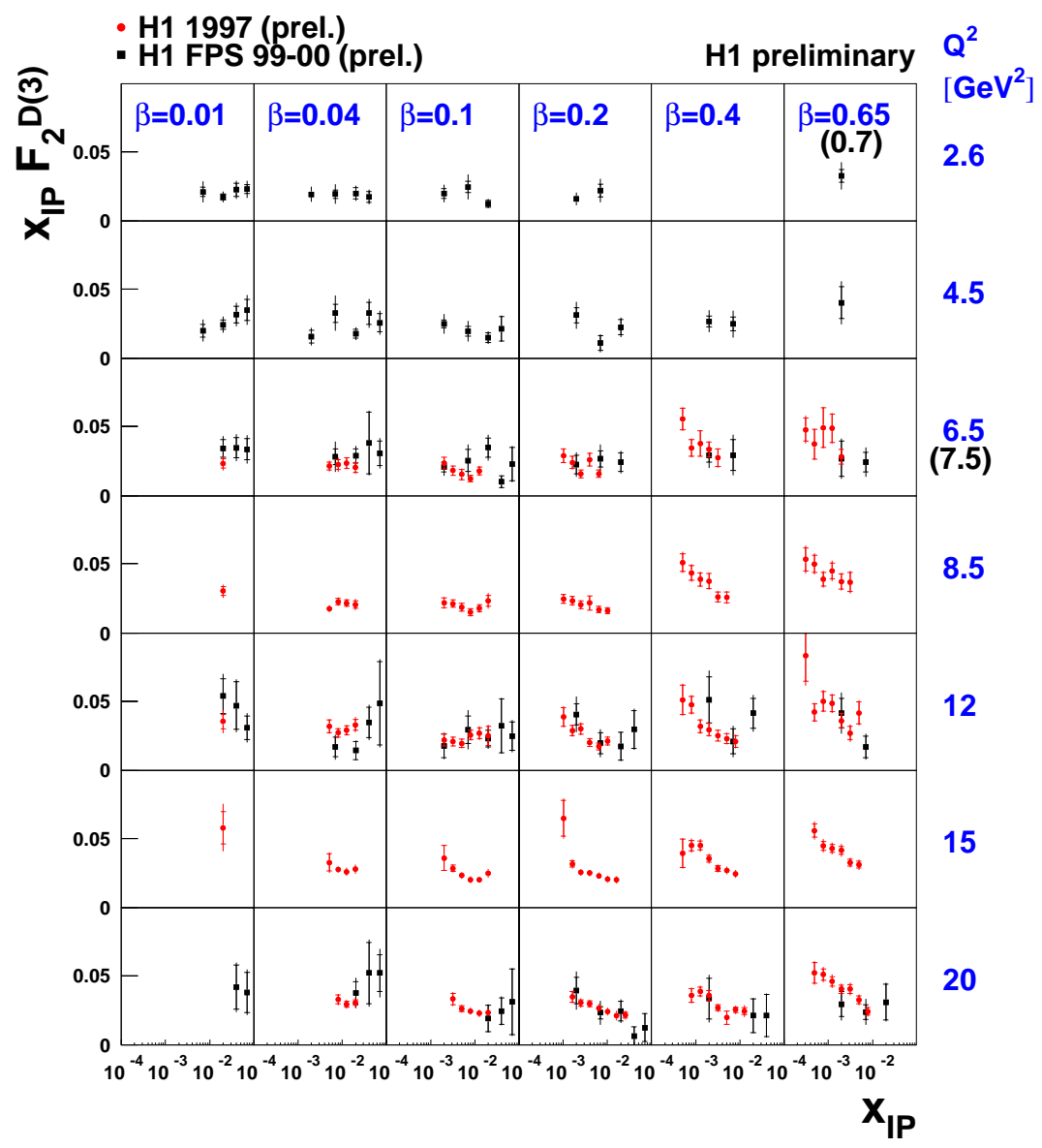

Figure 3: $x_{I P} F_{2}^{D(3)}$ obtained with the FPS is compared to the recent rapidity gap measurement by H1.

\subsection{Azimuthal asymmetry}

For unpolarised positrons and protons, the diffractive cross section can be decomposed as $d \sigma^{D} / d \Phi \propto \sigma_{T}^{D}+\epsilon \sigma_{L}^{D}-2 \sqrt{\epsilon(1+\epsilon)} \sigma_{L T}^{D} \cos \Phi-\epsilon \sigma_{T T}^{D} \cos 2 \Phi$, where $\Phi$ is the angle between the positron and proton scattering planes in the $\gamma^{*} p$ rest frame, $\sigma_{T}^{D}$ and $\sigma_{L}^{D}$ are the cross sections for transverse and longitudinal photons, $\sigma_{L T}^{D}$ and $\sigma_{T T}^{D}$ are interference terms and $\epsilon$ is the polarisation parameter. If $\sigma_{L T}^{D} \neq 0$, the $\phi$ distribution is non-uniform. A measurement of this non-uniformity would furthermore imply a non-zero value of $R^{D}=\sigma_{L}^{D} / \sigma_{T}^{D}$ [i $[\bar{i} \mid]$. Figure 15.' (left) shows $d \sigma^{D} / d \Phi$ as measured by the ZEUS collaboration together with a fit to the form $d \sigma^{D} / d \Phi \propto 1+A_{L T} \cos \Phi$ which yields $A_{L T}=-0.049 \pm 0.058(\text { stat. })_{-0.009}^{+0.056}($ syst. $)$, compatible with zero indicating that the interference term $\sigma_{L T}^{D}$ is small. Figure shows a first measurement of $A_{L T}$ in bins of $x_{I P}, \beta,-t$ and $Q^{2}$. Theoretical predictions for

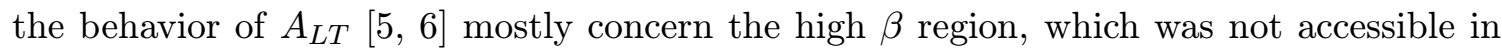
this analysis. No strong dependence of $A_{L T}$ on $x_{I P}, \beta, t$ and $Q^{2}$ is observed in the data.

\section{References}

[1] P. Newmanm, these proceedings.

[2] H1 Collaboration, Abstract 809, Paper submitted to EPS Conf. on HEP, Budapest 2001. 

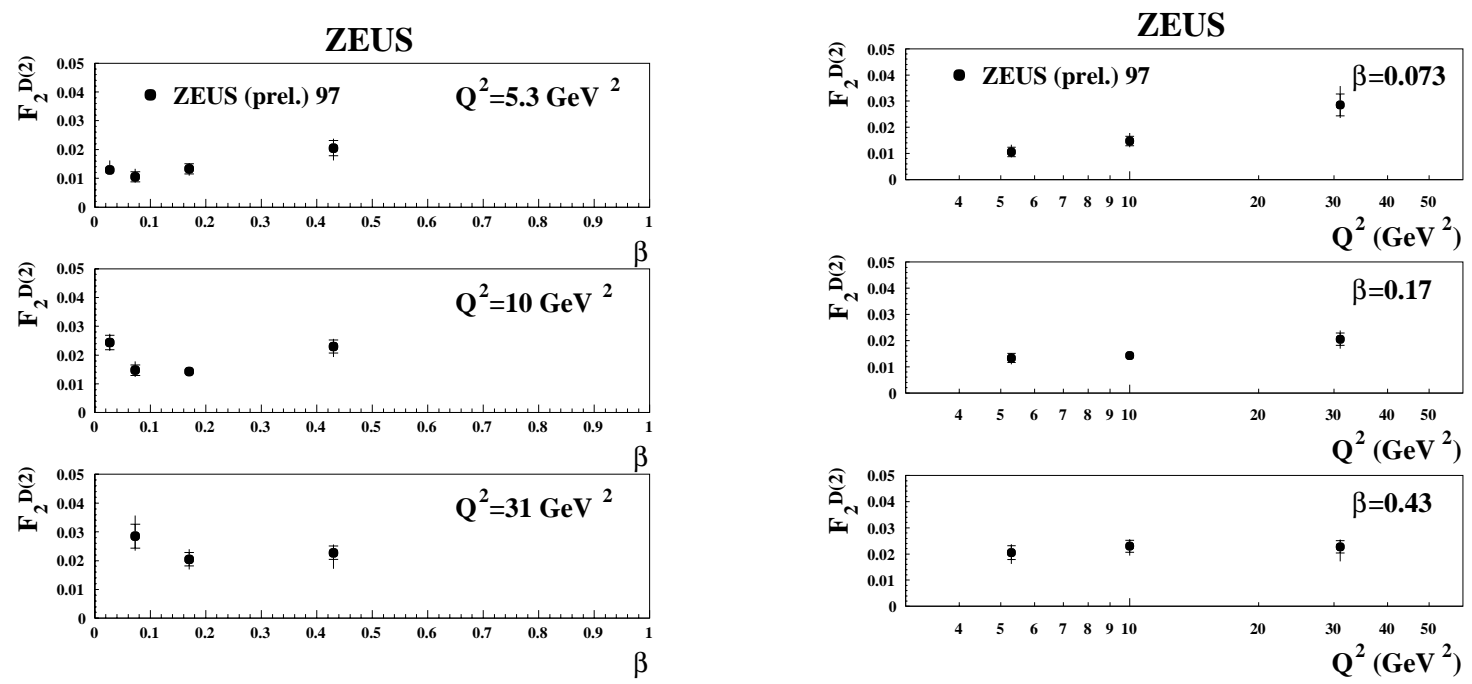

Figure 4: The values of $F_{2}^{D(2)}\left(\beta, Q^{2}\right)$, obtained from a fit to $F_{2}^{D}(3)$ are shown as a function of $\beta$ (Left) and $Q^{2}$ (Right).
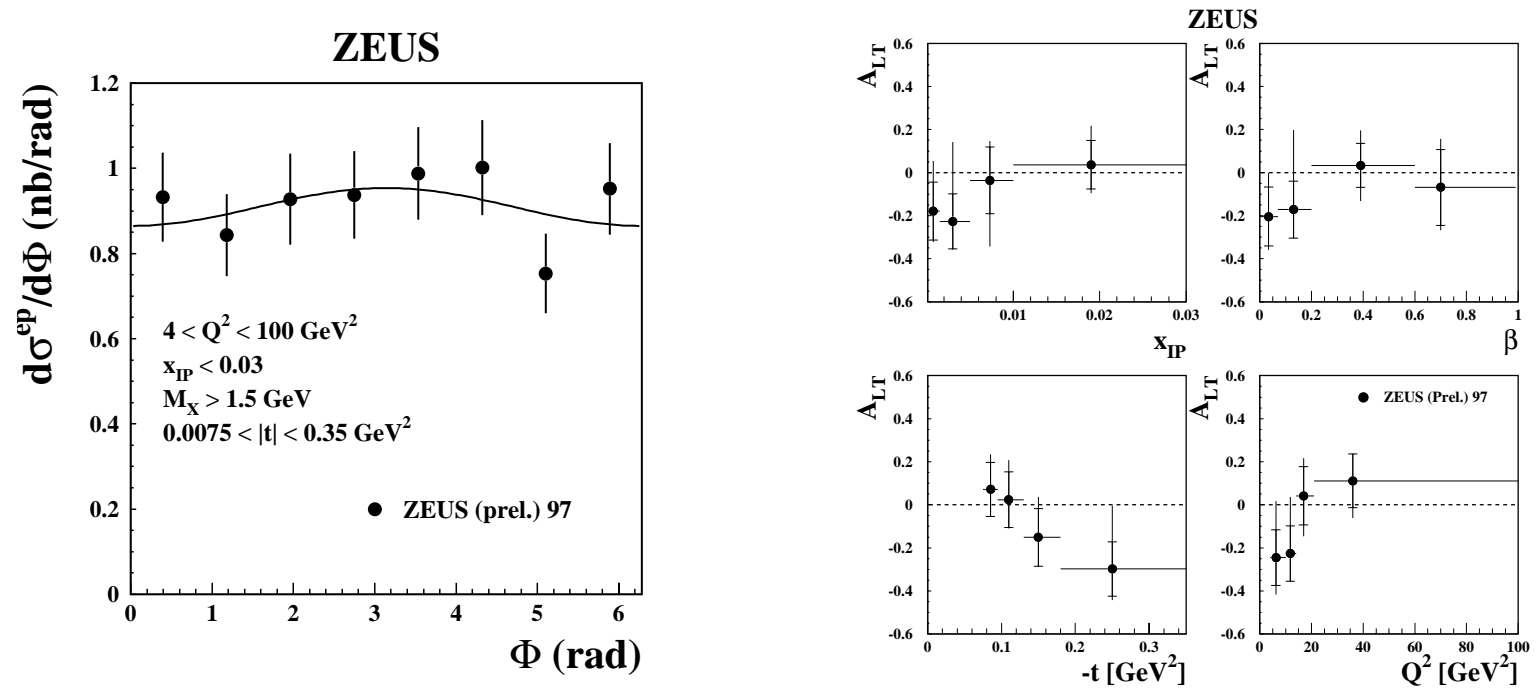

Figure 5: Left: The cross section $d \sigma / d \Phi$ together with the fit described in the text. Right: The azimuthal asymmetry $A_{L T}$ determined as a function of $x_{I P}, \beta, t$ and $Q^{2}$.

[3] ZEUS Collaboration, Abstract 566, Paper submitted to EPS Conf. on HEP, Budapest 2001.

[4] H1 Collaboration, Abstract 808, Paper submitted to EPS Conf. on HEP, Budapest 2001.

[5] N.N. Nikolaev et al., Phys, Rev. D 59, 091501 (1999).

[6] T. Arens et al., Z. Phys. C 74, 651 (1997); T. Gehrman and W.J. Stirling, Z. Phys. C 70, 89 (1996); N.N. Nikolaev and B.G. Zakharov, hep-ph/9706343. 